\title{
KLF8 promotes cancer stem cell-like phenotypes in osteosarcoma through miR-429-SOX2 signaling
}

\author{
L. ZHANG, P. YANG, Q. LIU, J. WANG, F. YAN, L. DUAN, F. LIN* \\ Department of Oncology, Affiliated Eighth People's Hospital, Jiangsu University, Shanghai, China \\ *Correspondence: linfengdoc@163.com
}

Received June 11, 2019 / Accepted September 4, 2019

\begin{abstract}
Krüppel-like factor 8 (KLF8) regulates critical gene transcription associated with different types of cancer. A novel paradigm in tumor biology suggests that the initiation and progression of osteosarcoma (OS) are driven by osteosarcoma stem cell-like cells (OSCs), but the role and underlying mechanisms of KLF8 in OSCs are poorly elucidated. In this study, an obviously increased level of KLF8 is shown in 9 out of 10 primary OS tissues and is associated with the poor progressionfree interval. Significantly, KLF8 expression in CD133+ OSCs is higher than that in CD133- counterparts. By knocking down KLF8 in CD133+ OSCs, we show that si-KLF8-OSCs can hardly form compact spheres. In the meantime, infection with si-KLF8 in CD133+ OSCs results in the downregulation of OCT4 and SOX2; increased adriamycin (ADM) sensitivity; and decreased tumorigenic potential in vivo. Mechanisms study demonstrates that KLF8 directly binds the miR-429 promoter region and regulates its expression transcriptionally. Furthermore, we indicate that miR-429 directly targets SOX2 to mediate cancer stem cell-like features in CD133+ OSCs. In the clinic, miR-429 levels are negatively associated with KLF8 levels in OS, suggesting that an elevated KLF8/miR-429 ratio may have clinical value as a predictive biomarker. In conclusion, targeting the KLF8-miR-429-SOX2 signaling pathway may provide an effective therapeutic approach to suppress the initiation and progression of OS.
\end{abstract}

Key words: Krüppel-like factor 8, osteosarcoma, cancer stem cell-like cells, miR-429, SOX2, CD133

Osteosarcoma (OS) is the third most common cancer in adolescence and is the most frequent appearance of primary bone tumors [1]. Despite the advanced intensive treatment, including a combination of surgery, radiotherapy and high dose chemotherapy [2], the 5-year survival and the outcome for OS patients presenting with metastasis have remained poor.

A rising number of studies indicate that cancer stem celllike cells (CSCs), characterized by increased tumorigenicity, self-renewal ability and multipotency, contribute to tumor initiation and progression [3,4]. CSCs have been observed in several types of tumors and have been critically linked to malignant transformation, and maintenance of cancers [4]. OS, as heterogeneous cancer, has been demonstrated to comprise of an array of distinguishable sub-clones [5], where osteosarcoma stem cell-like cells (OSCs) existed [6-8]. Thus, therapeutics targeting OSCs may be an effective strategy to prevent OS originating and progressing.

Krüppel-like factor (KLF8) shares homology in its three $\mathrm{C} 2-\mathrm{H} 2$ zinc finger DNA binding domains and was found to play a crucial role in different types of malignant tumors [9-12]. Despite the dual transcription factor function which transcriptionally activates or represses tumor-related genes, recently, it has emerged as an important cancer-promoting protein $[13,14]$. KLF8 is found highly overexpressed in some invasive human cancers, including breast cancer, ovarian cancer, etc. [14-16], and associated with a poor prognosis or an early recurrence. Our previous study demonstrated that KLF8 was upregulated and its knockdown in Saos-2 OS cells resulted in significant inhibition of proliferation and invasion [17]. However, the mechanisms underlying KLF8 in OS is poorly elucidated. In CSCs, recent studies show that KLF8 promotes the proliferation of hematopoietic stem cells, and stem cell populations in the kidney by regulating the transcription of Wnt signaling [16]. In addition, KLF8 is one of the master epithelial to mesenchymal transition (EMT)-promoting transcriptional factors [18]. Considering that a large body of evidence suggests a role of aberrantly activated EMT in CSC properties regulation $[19,20]$, these findings prompted us to investigate the role and mechanisms of KLF8 in OSCs. 
MicroRNAs (miRNAs) are a family of short non-coding regulatory RNAs that can bind to the $3^{\prime}$-untranslated region (3'UTR) of target mRNAs to induce translation repression or mRNA cleavage and to attenuate protein expression [21]. miRNAs have emerged as important regulators in either tumor suppression or promotion [22]. miR-429 was reported suppressing the progression and metastasis of OS by targeting ZEB1, one of the most crucial EMT factors [23, 24], which suggests that miR-429 may regulate the EMT-associated CSC properties. Both of miR-429 and KLF8 are the crucial EMT factors in OS, however, whether they have any underlying links is unknown. In the meantime, the miR- 429 regulation of EMT-associated CSC properties remains unclear.

Here, we aim to explore the role and underlying mechanisms of KLF8 in OSCs. In this study, an obviously increased level of KLF8 is showed in 9 out of 10 primary OS tissues and is associated with the poor progression-free interval. Significantly, the KLF8 expression in CD133+ OSCs is higher than that in $\mathrm{CD}^{-133^{-}}$counterparts. By knocking down KLF8 in CD133+ OSCs, we show that si-KLF8-OSCs can hardly form compact spheres. In the meantime, $\mathrm{CD}_{133^{+}}$OSCs infected with si-KLF8 result in the downregulation of OCT4 and SOX2, increased ADM sensitivity, and decreased tumorigenic potential in vivo. Mechanisms studies demonstrate that KLF8 directly binds the miR- 429 promoter region and regulates its expression transcriptionally. Furthermore, we indicate that miR-429 directly targets SOX2 to mediate cancer stem celllike features in $\mathrm{CD} 133^{+}$OSCs.

\section{Materials and methods}

Animal and cell culture. Female athymic BALB/c nu/ nu mice, 3-4 weeks old, obtained from HFK Bioscience (China), were maintained at the Animal Core Facility at Affiliated Eighth People's Hospital, Jiangsu University, under specific pathogen-free (SPF) condition. All studies on mice were conducted in accordance with the National Institutes of Health 'Guide for the Care and Use of Laboratory Animals' and were approved by the ethics committee of Affiliated Eighth People's Hospital, Jiangsu University. The human gastric cancer cell line MG-63 and U-2 were purchased from ATCC (Manassas, USA) and were properly kept in Affiliated Eighth People's Hospital, Jiangsu University. All the cell lines were authenticated by Short Tandem Repeat-Polymerase Chain Reaction (STR-PCR).

Sample collection. 10 patients with primary osteosarcoma in Affiliated Eighth People's Hospital, Jiangsu University were enrolled in this study from January 2013 to June 2016. Informed consent for the additional core-needle biopsy and experimental use of tumor samples was obtained from all patients, following a protocol approved by the Ethics Committee of Affiliated Eighth People's Hospital, Jiangsu University.

Flow cytometry assay and fluorescence activated cell sorting (FACS). Flow cytometry assay was done on single- cell suspensions obtained by enzymatic digestion of spheres which were derived from primary colorectal cancer samples and labeled with FITC conjugated anti-CD133 antibody (Miltenyi Biotec, Germany) by using an Epics Altra flow cytometer (Beckman Coulter, USA).

Spheres derived from primary tumor samples were dissociated into single cells by enzymatic digestion. Single-cell suspensions were washed and incubated in staining solution $1 \%$ BSA and $2 \mathrm{mM}$ EDTA with the specific antibodies at appropriate dilutions. Then the cells were sorted to $\mathrm{CD} 133^{+}$ and $\mathrm{CD} 133^{-}$subpopulations.

Western blot analysis. Cells were harvested and lysed in RIPA lysis buffer ( $150 \mathrm{mM} \mathrm{NaCl}, 50 \mathrm{mM}$ Tris- $\mathrm{HCl}$ (pH 8.0), $0.1 \%$ SDS, 2 mM EDTA, 1 mM PMSF, 1\% NP40, $5 \mu \mathrm{g} / \mathrm{ml}$ aprotinin, and $1 \mu \mathrm{g} / \mathrm{ml}$ leupeptin) on ice, and then centrifuged at $10000 \times \mathrm{g}$ for $10 \mathrm{~min}$. Total proteins were resolved by $12 \%$ SDS-PAGE (Bio-Rad Laboratories, Inc, Hercules, CA, USA) and blotted onto nitrocellulose membranes (Amersham Biosciences Corp., Pittsburgh, PA, USA). Membranes were blocked with $10 \%$ non-fat milk powder at room temperature for $2 \mathrm{~h}$ and incubated overnight with primary antibodies purchased from Abcam, Cambridge, USA: anti-KLF8 (ab168527, 1:1000), anti-OCT4 (ab184665, 1:2000), anti-SOX2 (ab79351, 1:1500) or from SigmaAldrich, Louis, USA: anti- $\beta$-actin antibody (A1978, 1:2000). After three 5 min washes in Triethanolamine-Buffered Saline solution with $0.1 \%$ Tween-20 (TBS-T), membranes were incubated with horseradish peroxidase (HRP) conjugated secondary antibodies (sc-2357, 1:2000; Santa Cruz Biotechnology, Dallas, USA) for $4 \mathrm{~h}$ at room temperature and then washed again in TBS-T and visualized with an enhanced chemiluminescence kit (ECL-kit, Santa Cruz Biotechnology, Dallas, TX, USA). All experiments were performed in triplicate.

Animal studies and bioluminescence. All of the procedures were carried out according to the animal protocol approved by the Age-matched female mice were used. A total of $3 \times 10^{5}$ luciferase-expressing si-KLF8 cells, si-NC cells were subcutaneously injected. Tumor progression was monitored using bioluminescence (IVIS Spectrum), and survival was monitored daily. Tissue samples were collected on the indicated days after injection for pathological analysis.

Luciferase assays. Luciferase reporter assays were carried out using the Luciferase Assay System (Promega). The reporter gene construct (pGL3 Luciferase Reporter vector, Cat. E1741, Promega) and pTK-Renilla construct (pTK-Green Renilla Luc vector, Cat. 16154, Thermo Scientific) were co-transfected into cells, and luciferase activity was measured with the Dual-Luciferase Reporter Assay System (Promega). The results are expressed as luciferase/ renilla ratios and represent the average \pm s.d. of at least three experiments, each performed in triplicate.

Quantitative real-time PCR analysis (qPCR). Realtime PCR was performed using the Applied Biosystems ${ }^{\circ}$ StepOne $^{\text {Twx }}$ Real-Time PCR Systems (Applied Biosystems). 
All qPCR reactions were performed in duplicate and the amplification signal from the target gene was normalized to a GAPDH signal. For the detection of mature miR-429, the TaqMan MicroRNA assay kit (Applied Biosystems) was used according to the manufacturer's instructions. All qPCR reactions were performed in duplicate and the amplification signal from the target miRNA was normalized to a U6 signal. The average of three experiments each performed in triplicate with standard errors is presented. Primers are as following: KLF8, F: 5'-TTCAGAAGGTGGCTCAATGC-3', R: 5'-GGAGTGTTGGAGAAGTCATATTAC-3'; GAPDH, F: 5'-TGACTTCAACAGCGACACCCA-3', R: 5'-CACCCTGTTGCTGTAGCCAAA-3'.

Promoter assays. As for the promoter assays, for analyses of the miR-429 promoters, pGL3 reporter plasmids containing the miR-429 regulatory sequences fused to luciferase cDNA were used. Co-transfection with b-galactosidase reporter and detection of relative luciferase units.

MTT assay. The MTT assay (Sigma Aldrich, USA) was used to determine relative cell growth every $24 \mathrm{~h}$ for cell growth curves. A total of $1 \times 10^{4}$ cells $/ \mathrm{ml}$ were plated into 96-well plates, incubated at $37^{\circ} \mathrm{C}$ and cultured overnight. $20 \mu \mathrm{l}$ of $5 \mathrm{mg} / \mathrm{ml} \mathrm{MTT}$ was added to the media for $4 \mathrm{~h}$ incubation at $37^{\circ} \mathrm{C}$. Following removal of the culture medium, the remaining crystals were dissolved in $150 \mu$ DMSO (Sigma Aldrich, USA). Absorbance (A) was measured spectrophotometrically in a microplate reader (Bio-Rad, USA) at a wavelength of $490 \mathrm{~nm}$. The curve of growth was drawn with the absorbance (A) measured spectrophotometrically in a microplate reader (Bio-Rad, USA) at a wavelength of $490 \mathrm{~nm}$.

Statistical analysis. Unless otherwise explained specifically, all results were expressed as mean \pm SEM and analyzed using the SPSS 20.0 software (SPSS, Chicago, IL, USA). Differences between groups were assessed using the Student's t-test. The relationship between SLC34A2 expression and the clinicopathological features of gastric cancer was analyzed by Pearson's $\chi^{2}$ test. The overall survival of the two patient groups was analyzed by the log-rank test using the Kaplan-Meier method. A $p<0.05$ was considered statistically significant.

\section{Results}

KLF8 is overexpressed in CD133 ${ }^{+}$OSCs and primary OS tissues. CD133 serves as the putative marker for different types of CSCs [25]. Flow cytometry showed the presence of a variable fraction of CD133 positive cells, ranging from $0.03 \%$ to $1.83 \%$, in 10 out of 10 OS specimens (Supplementary Table S1). We sorted both $\mathrm{CD} 133^{+}$and CD133- cells from MG-63 and U-2 -two OS cell lines- by FACS (Figure 1A). After 2-4 weeks, by serum-free cultivation, CD133 ${ }^{+}$cells were able to form compact self-renewing spheres in serumfree medium, whereas $\mathrm{CD} 133^{-}$cells could not form compact spheres (Figure 1B). In the meantime, we observed an increased level of KLF8 in 9 out of 10 primary tumor tissues compared with their adjacent counterparts (Supplementary
Table S1). Overexpression of KLF8 was obviously associated with the reduced progression-free interval (Figure 1C). Concurrently, the expression of KLF8 in CD133+ OSCs was much higher than that in $\mathrm{CD}_{133^{-}}$counterparts (Figures 1D, $1 \mathrm{E})$.

KLF8 promotes cancer stem cell-like features of $\mathrm{CD} 133^{+}$ OSCs in vitro and in vivo. To evaluate the role of KLF8 in cancer stem cell-like features maintaining, we constructed $s i-K L F 8$ stable cell lines by a lentivirus-mediated method. We found that si-KLF8-OSCs could hardly form compact spheres compared with the $s i$-NC-OSCs group and non-treated OSCs group (Figure 2A). si-KLF8 transfection in $\mathrm{CD} 133^{+}$OSCs resulted in increased cisplatin sensitivity: si-KLF8 substantially enhanced cell death of $\mathrm{CD}_{133^{+}} \mathrm{OSCs}$ in the presence of adriamycin (ADM) (Figure 2B). Additionally, the expression levels of OCT4 and SOX2 were obviously decreased in si-KLF8-OSCs compared with those in other control groups (Figure 2C).

In vivo, si-KLF8-OSCs, si-NC-OSCs and non-treated OSCs were injected by a subcutaneous route. Tumor formation of the $s i$-KLF8-OSCs group was lower and in decreased tumor take compared with that observed in another group (Figures 2D, 2E). To investigate the long-term tumorigenic potential, we performed serial transplantation assays of cells isolated from tumor xenografts originally derived from $s i-K L F 8$-OSCs, si-NC-OSCs and non-treated OSCs injection into nude mice. Cells derived from non-treated OSCs and $s i$-NC-OSCs tumors were able to generate xenografts in primary, secondary, and tertiary transplantation. Whereas cells from si-KLF8-OSCs tumors lost tumorigenic potential during the tertiary transplantations (Figure 2F).

miR-429 is regulated by KLF8 directly. The expression of miR-429 was found reduced in OS tissues compared with that in adjacent normal counterparts (Supplementary Table S1). We further found that $s i-K L F 8$ in CD $133^{+}$OSCs resulted in a remarkable reduction of mature miR-429 expression (Figure 3A). To further define the transcriptional regulation of miR-429, its promoter region containing numerous putative KLF8 binding sites was constructed. Luciferase reporter assay showed that the KLF8 knockdown significantly inhibited miR-429 promoter activity, while the overexpression of KLF8 enhanced the luciferase activity (Figure 3B). These data suggest that KLF8 directly binds to the miR-429 promoter region and regulates its expression transcriptionally.

miR-429 directly targets SOX2 that is negatively correlated with miR-429 level. 3'UTR of SOX2 was indicated as one of the putative miR-429 targets by online prediction software, including TargetScan 6.2 and PicTar (Figure 4A). To further confirm whether SOX2 is the direct target of miR-429, luciferase reporter assays were performed on OSCs transfected with wild type or mutated SOX2 3'UTR. As shown in Figure 4B, the luciferase activity in OSCs transfected with wild type SOX2 3'UTR was significantly reduced in the Lv-miR-429 group. On the contrary, the effect of Lv-miR-429 

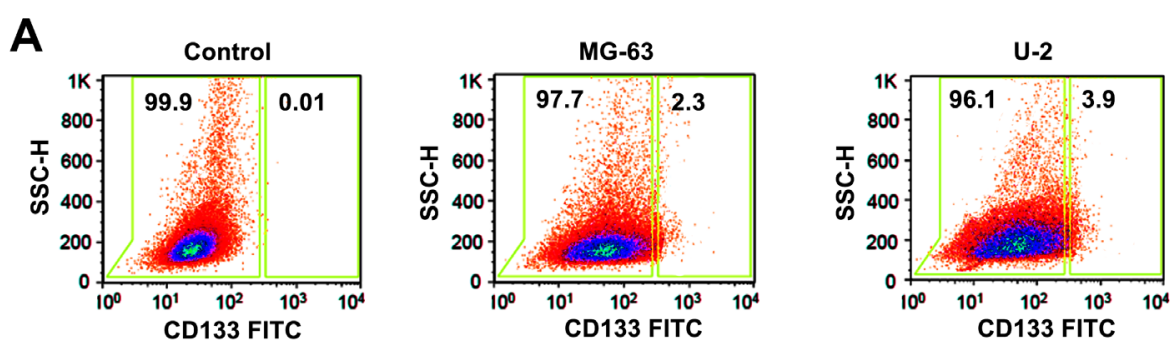

B
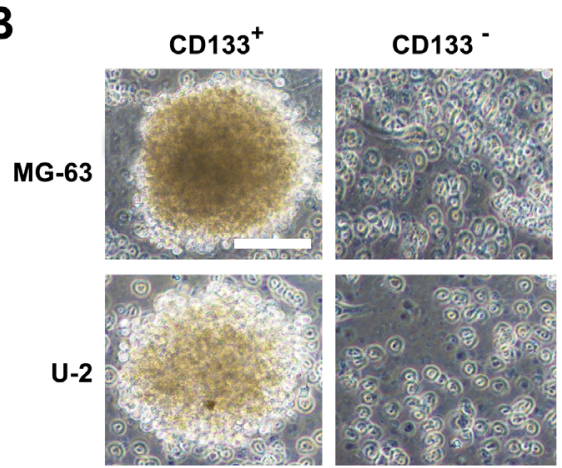

C

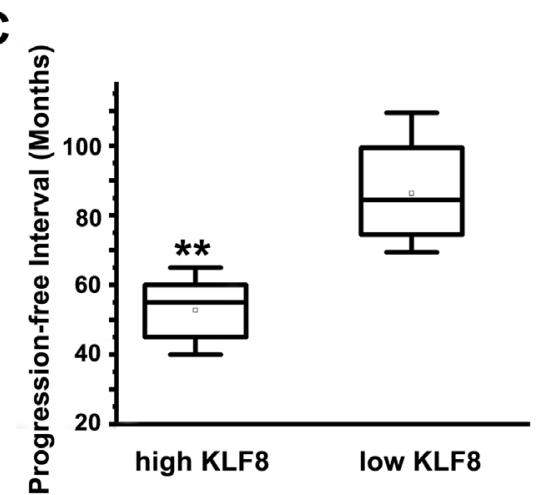

D

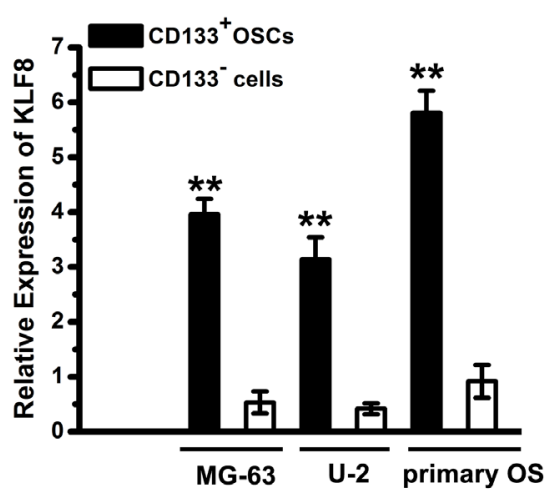

$\mathbf{E}$

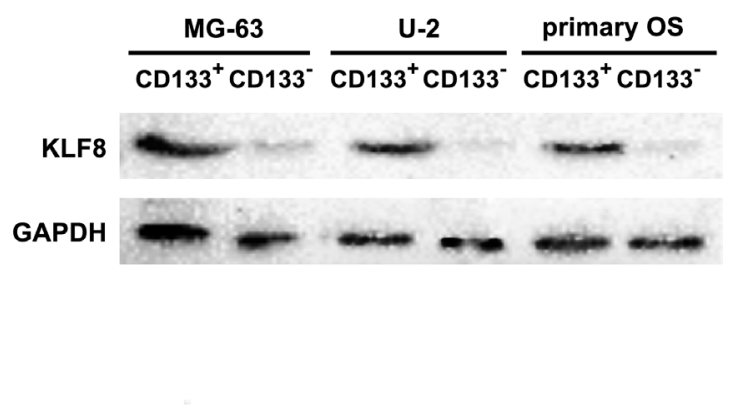

Figure 1. KLF8 is overexpressed in CD133+ ${ }^{+}$OSCs and primary OS tissues. A) CD133+ fractions in MG-63 and U-2 cell line cells by FACS. B) Phasecontrast images of $\mathrm{CD}_{133^{+}}$sphere cells and $\mathrm{CD} 133^{-}$cells. Scale bar, $50 \mu \mathrm{m}$. C) Comparison of the progression-free intervals in OS patients with high or low expression of KLF8. The standard is according to the medium relative expression of KLF8 in these 10 OS patients. Note: Columns, mean of three individual experiments; $S^{* *} p<0.01$. D) Relative expression of KLF8 in CD133+ OSCs and CD133- cells. Primary OS is from case 7 tissues. Note: Columns, mean of three individual experiments; SD, ${ }^{* *} \mathbf{p}<0.01$. E) Immunoblotting KLF8 expression on monolayer or 3-dimensional cultures. GAPDH was used as a loading control.

was completely abrogated in the mutant construct. Moreover, by investigating the correlation of miR-429 and SOX 2 in OS patient cohorts, miR-429 levels were observed declined in CD133+ OSCs compared with those in CD133- counterparts, while the trend of SOX2 expression was opposite (Figure 4C), confirming that SOX2 is negatively correlated with miR-429 level.

\section{Discussion}

A better understanding of how the tumors originate and progress is needed for the development of targeted therapies for both primary and metastatic OS. Considering the poten- tial role of OSCs for OS diagnosis and treatment, clarifying the regulatory mechanism of OSCs is crucial for the development of novel treatment options. Our study provides insight for the role of KLF8 involving the promotion of OSCs stem cell-like phenotypes through the miR-429-SOX2 signaling pathway.

KLF8 was recently emerged as crucial cancer-promoting gene [26]. It was found highly overexpressed in some invasive human cancers, including breast cancer, ovarian cancer, etc. [14-16], and associated with a poor prognosis or an early recurrence. However, the expression and the role of KLF8 had been unclear in OS, until we showed that KLF8 was upregulated in OS and knockdown of KLF8 in 
A

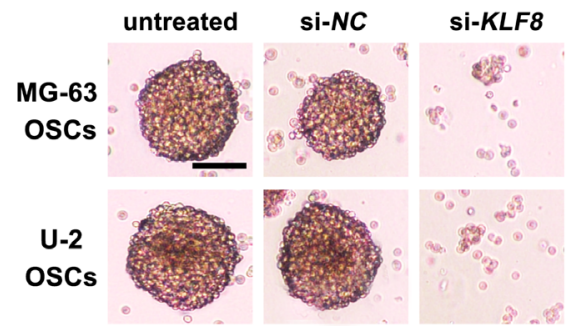

C

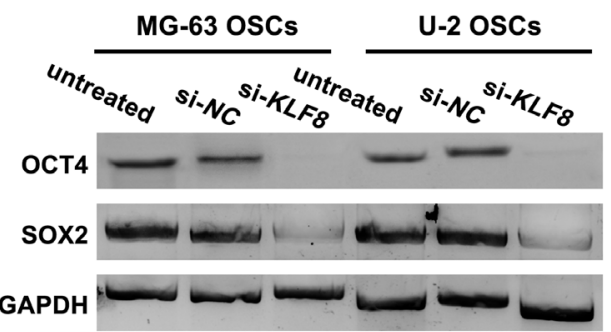

D

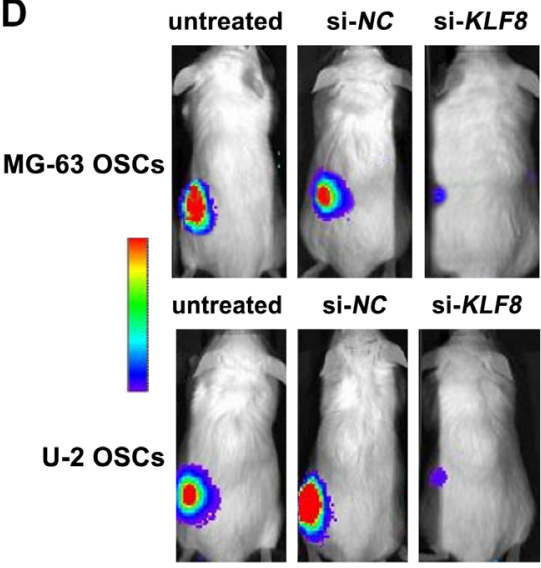

$\mathbf{F}$

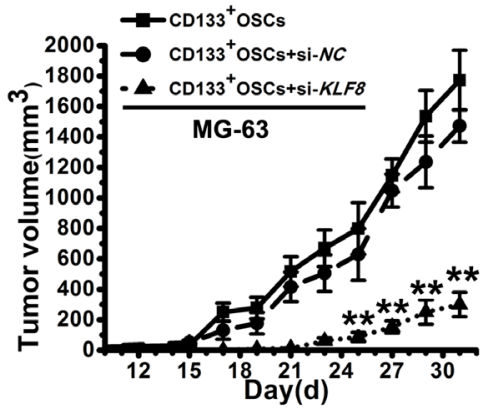

B $\quad 100 \rightarrow-$ CD $133^{*}$ OsCs
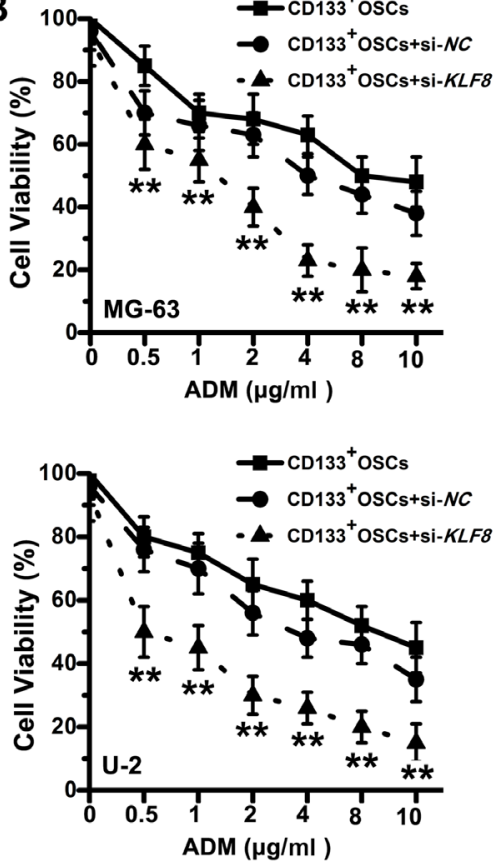

$\mathbf{E}$
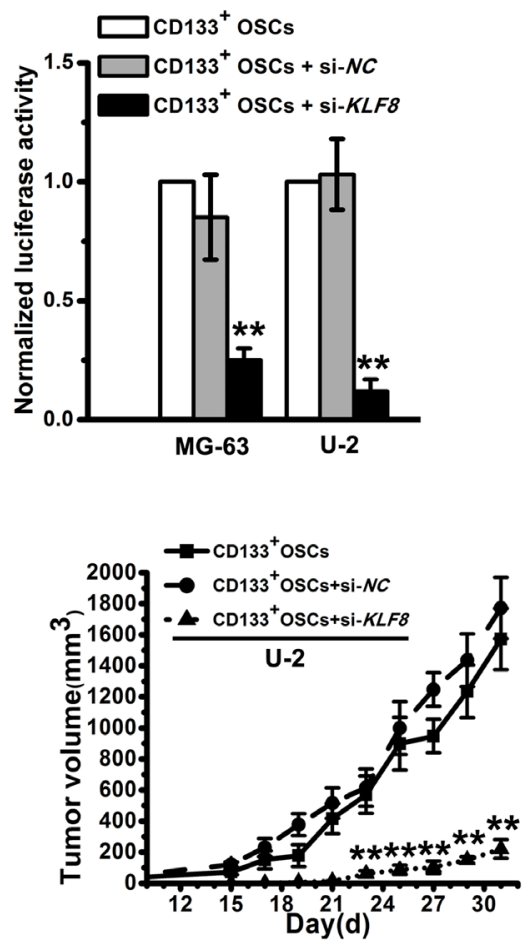

Figure 2. KLF8 promotes cancer stem cell-like features of $\mathrm{CD}_{133}{ }^{+} \mathrm{OSCs}$ in vitro and in vivo. A) Phase-contrast images of CD133 ${ }^{+} \mathrm{OSCs}_{\text {treated }}$ with si-KLF8 or si-NC. Scale bar, $50 \mu \mathrm{m}$. B) With increasing concentrations of doxorubicin (ADM) from $0.5 \mathrm{to} 10 \mu \mathrm{g} / \mathrm{ml}$, the percentage of viable cells of $\mathrm{CD} 133^{+}$OSCs infected with si-KLF8 or si-NC were measured by MTT. Note: Columns, mean of three individual experiments; $\mathrm{SD}^{\star *} \mathrm{p}<0.01$. C) Westernblotting was used with antibodies specific for OCT4 and SOX2 in $\mathrm{CD}_{133}{ }^{+} \mathrm{OSCs}$ with or without infection of si-KLF8 or si-NC. GAPDH was used as the control. D) Bioluminescence imaging and luciferase activity of mice with subcutaneous injection of CD133+ ${ }^{+}$OSCs with or without infection of si-KLF8 or si-NC. E) The luciferase activity normalized with that of control at day 21 . Note: Columns, mean of three individual experiments; $S D{ }^{\star *} p<0.01$. The number of mice for each cohort is 3 . F) In vivo serial transplantation assay. A total of $1 \times 10^{5} \mathrm{CD}_{133^{+}}$OSCs with or without infection of si-KLF8 or si$N C$ were injected s.c. into nude mice. Derived tumor xenografts were dissociated to single-cell suspension and then serially re-injected in mice $\left(1 \times 10^{5}\right.$ cells), generating secondary and then tertiary tumors. Tumor growth curves of primary and tertiary tumors are shown. Note: Columns, mean of three individual experiments; $\mathrm{SD}^{* *} \mathrm{p}<0.01$. 
A
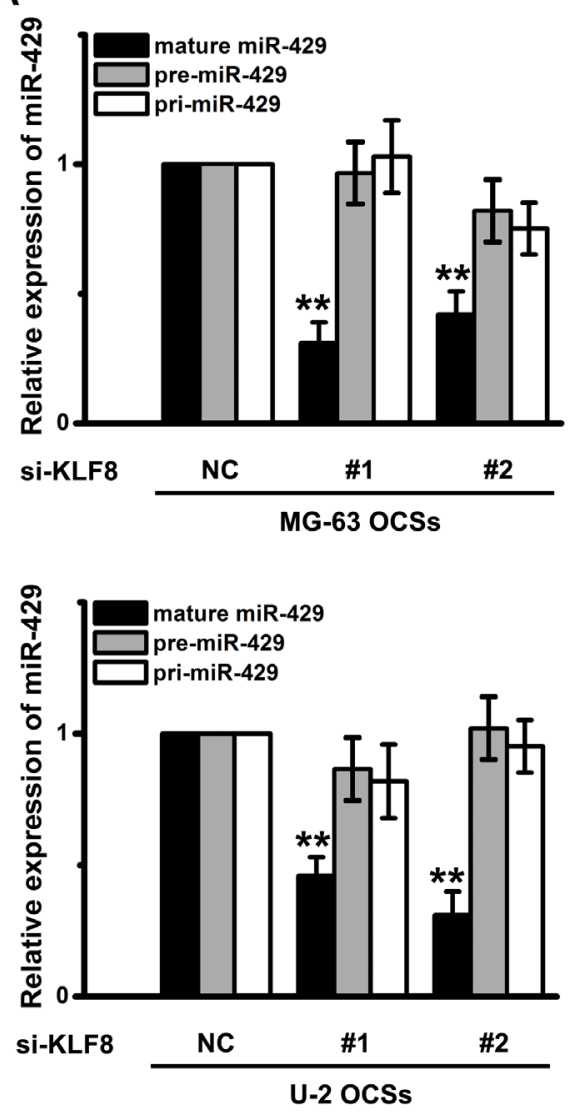

B

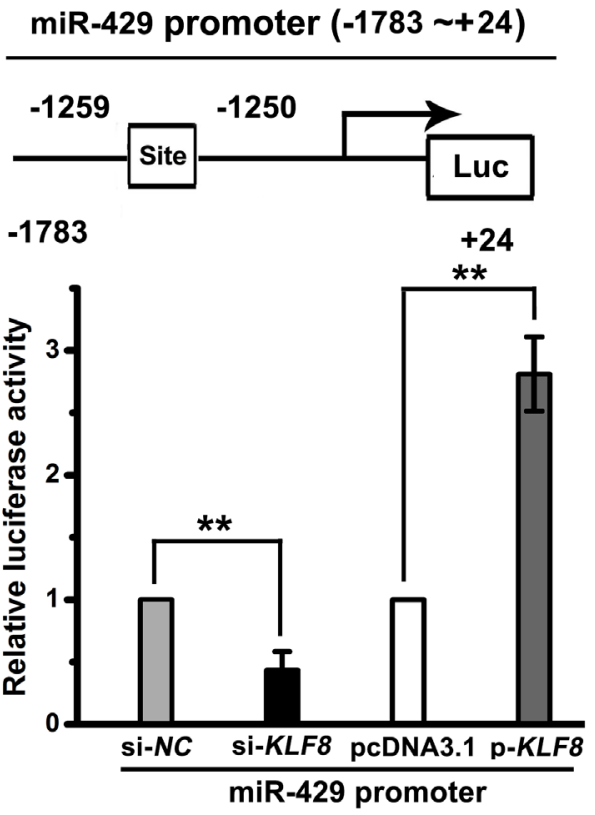

Figure 3. miR-429 is regulated by KLF8 directly. A) The expression of mature miR-429, pre-miR-429, and pri-miR-429 was detected in CD133 ${ }^{+}$OSCs with or without infection of si-KLF8 (\#1/\#2 mean different si-KLF8-OSCs clones) by qPCR. Note: Columns, mean of three individual experiments; SD ${ }^{* *} \mathrm{p}<0.01$. B) The diagram shows miR-429 and its putative binding site at the 3'UTR of KLF8 and the sequences of WT and MT KLF8 3'UTR. A luciferase reporter assay was used to assess whether miR-429 can directly bind to the 3'UTR of KLF8. Note: Columns, mean of three individual experiments; $\mathrm{SD}^{\star *} \mathrm{p}<\mathbf{0 . 0 1}$.

Saos-2 OS cells resulted in significant inhibition of proliferation and invasion [17]. KLF8 was demonstrated as the downstream effector of a focal adhesion kinase (FAK) [27]. FAK mediates human carcinoma progression, including cell proliferation, survival, migration, invasion, and EMT in many types of invasive human cancer [28, 29]. Considering that a large body of evidence suggests a role of activated EMT in CSC properties regulation, we speculate that KLF8 would be a potential target for OSCs wiping out. Here, we observed an increased level of KLF8 in primary tumor tissues compared with adjacent counterparts and the overexpression of KLF8 was remarkably associated with the reduced progression-free interval. Significantly, the expression of KLF8 in CD133+ OSCs was higher than that in CD133- counterpart. These data suggest that KLF8 may contribute to the maintaining of stem cell-like features in OSCs. Further biological function study showed that si-KLF8-OSCs could hardly form compact spheres, which is the hallmark of CSCs. si-KLF8 infection also resulted in increased cisplatin sensitivity, decreased the expression levels of OCT4 and SOX2, and reduced the tumorigenic potential in vivo. These results indicate that targeting KLF8 may provide an effective therapeutic approach to suppress the tumorigenicity of OSCs.

miR-429 was reported to suppress the progression and metastasis of OS by targeting ZEB1, one of the most crucial EMT factors [23, 24], which suggests that miR-429 may regulate the EMT-associated CSC properties. Both miR-429 and KLF8 are the crucial EMT factors in OS, however, whether they have any underlying links is unknown. In the mechanism studies, we showed that KLF8 directly bond to the miR-429 promoter region and regulated its expression transcriptionally. In addition, we found that the expression of miR-429 was declined in CD133+ OSCs and associated with poor overall survival. These findings are in line with the reports which showed that miR- 429 served as a tumor suppressor $[23,24]$.

Moreover, we demonstrated that SOX2 is the direct target of miR-429. SOX2, belonging to the SOX family, is a transcription factor that is important in the development and 


\section{A Position 477-483 of SOX2 3'UTR 5'...CAAgCAacuUuUguacaguaUuU... \\ has-miR-429 \\ UGCCAAAAUGGUCUGUCAUA IIIIII \\ SOX2 mutant

B
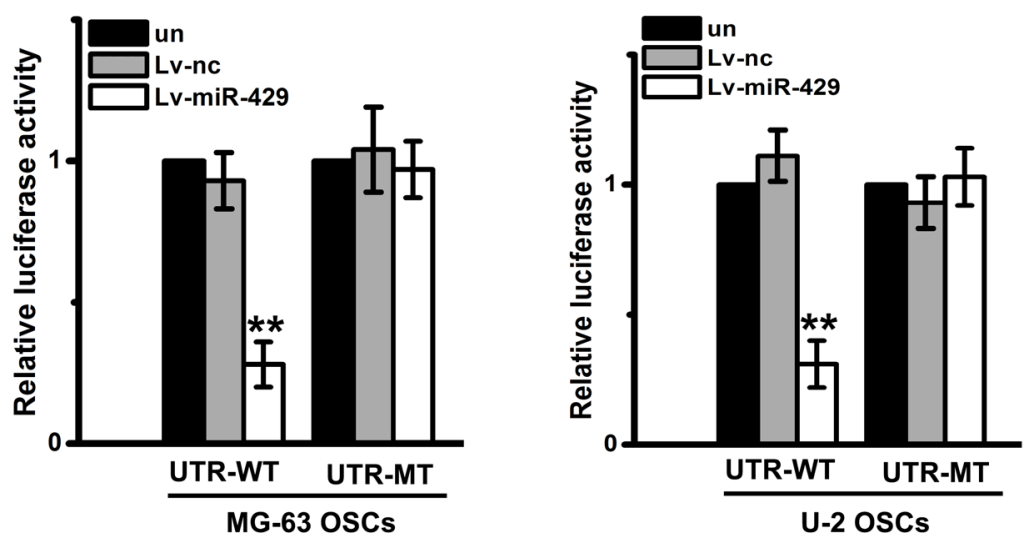

C
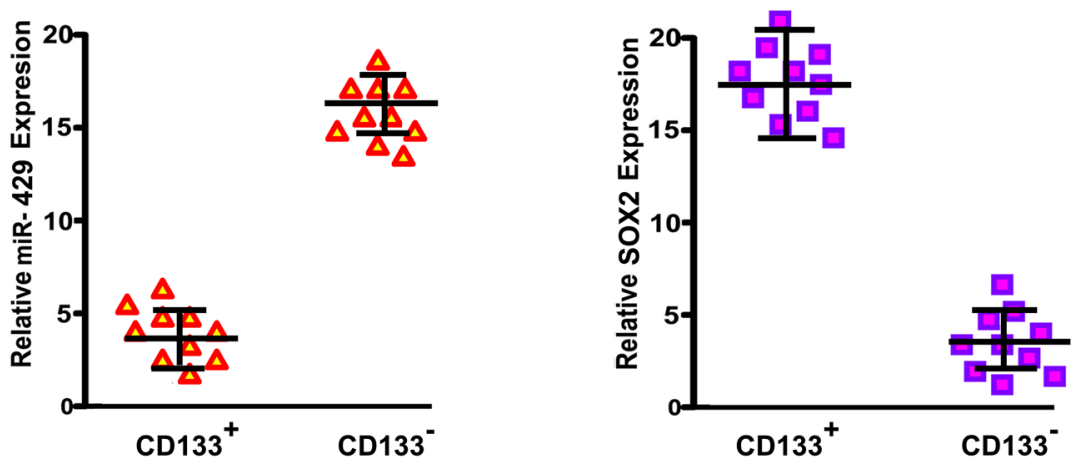

Figure 4. miR-429 directly targets SOX2 and SOX2 was negatively correlated with miR-429 level in OS tissues. A) Illustration of SOX2 3'UTR as well as the seed sequence of miR-429 showed the predicted target region on the 3'UTR of SOX2 mRNA. B) Dual-luciferase reporter assay with co-transfection SOX2 3'UTR plasmids and miR-429 mimics. The relative luciferase activity was obtained by firefly luciferase activity normalized against Renilla luciferase activity. Note: Columns, mean of three individual experiments; $S D{ }^{\star *} \mathbf{p}<0.01$. C) Downregulation of miR-429 was observed in OS tissues compared with that in adjacent ones by qPCR (left). Upregulation of SOX2 was observed in OS tissues compared with that in adjacent ones by qPCR (right). Note: Columns, mean of three individual experiments; $S D^{* *} \mathrm{p}<0.01$.

maintenance of the stem cell states [30, 31]. Together with OCT4 and NANOG, SOX2 forms the pluripotency regulatory network. SOX2 is associated with cancer progression by promoting the migration, invasion, and proliferation of cancer cells [32]. The stem cell transcription factor (Sox2) has been shown to maintain OSCs. Basu-Roy et al. [33] showed that SOX2 was required for OS cells self-renewal and that SOX2 antagonized the pro-differentiation. They imply that rather than be a canonical OS oncogene, SOX2 is essential for self-renewal of osteoblast precursors, which confirms our findings that KLF8 regulates miR-429-SOX2 axis to maintain the CSC features in $\mathrm{CD}_{133^{+}}$OSCs. On the other hand, Liu et al. [24] and Deng et al. [23] showed that miR-429 targets the EMT inducer ZEB1 to suppress the progression and metastasis of osteosarcoma. Combined with our report, we further imply that miR-429 mediates both SOX2 and ZEB1 to promote the EMT-associated CSC features, which attaches importance of the KLF8-targeted therapy for OS.

In conclusion, our findings indicate that KLF8 plays a crucial role in the maintenance of cancer stem cell-like features in $\mathrm{CD}_{133^{+}}$OSCs. Thus, targeting the KLF8-miR429-SOX2 signaling pathway may provide an effective therapeutic approach to suppress the initiation and progression of OS. Additionally, an elevated KLF8/ miR-429 ratio may have clinical value as a predictive biomarker in OS.

Supplementary information is available in the online version of the paper. 
Acknowledgements: This work was supported by the Shanghai Xuhui District Medical Peak Subject Project (SHXH201710) and Xuhui District Medical Science and Technology Project (SHXH201709, SHXH201709).

\section{References}

[1] RAYMOND AK, JAFFE N. Osteosarcoma multidisciplinary approach to the management from the pathologist's perspective. Cancer Treat Res 2009; 152: 63-84. https://doi. org/10.1007/978-1-4419-0284-9_4

[2] POGRIBNY IP, RUSYN I. nvironmental toxicants, epigenetics, and cancer. Adv Exp Med Biol 2013; 754: 215-232. https://doi.org/10.1007/978-1-4419-9967-2_11

[3] YOON C, PARK DJ, SCHMIDT B, THOMAS NJ, LEE HJ et al. CD44 expression denotes a subpopulation of gastric cancer cells in which Hedgehog signaling promotes chemotherapy resistance. Clin Cancer Res 2014; 20: 3974-3988. https:// doi.org/10.1158/1078-0432.CCR-14-0011

[4] ZHANG X, HUA R, WANG X, HUANG M, GAN L et al. Identification of stem-like cells and clinical significance of candidate stem cell markers in gastric cancer. Oncotarget. 2016; 7: 9815-9831. https://doi.org/10.18632/oncotarget.6890

[5] KOVAC M, BLATTMANN C, RIBI S, SMIDA J, MUELLER NS et al. Exome sequencing of osteosarcoma reveals mutation signatures reminiscent of BRCA deficiency. Nat Commun 2015; 6: 8940. https://doi.org/10.1038/ncomms9940

[6] SHIOZAWA Y, BERRY JE, EBER MR, JUNG Y, YUMOTO $\mathrm{K}$ et al.The marrow niche controls the cancer stem cell phenotype of disseminated prostate cancer. Oncotarget. 2016; 7: 41217-41232. https://doi.org/10.18632/oncotarget.9251

[7] YANG M, YAN M, ZHANG R, LI J, LUO Z Side population cells isolated from human osteosarcoma are enriched with tumor-initiating cells. Cancer Sci 2011; 102: 1774-1781. https://doi.org/10.1111/j.1349-7006.2011.02028.x

[8] WANG L, PARK P, ZHANG H, LA MARCA F, LIN CY. Prospective identification of tumorigenic osteosarcoma cancer stem cells in OS99-1 cells based on high aldehyde dehydrogenase activity. Int J Cancer 2011; 128: 294-303. https://doi. org/10.1002/ijc. 25331

[9] ALLEYNE GA. Toward a taxonomy of technical cooperation in health. Bull Pan Am Health Organ 1991; 25: 356-366.

[10] YAN Q, ZHANG W, WU Y, WU M, ZHANG M et al. KLF8 promotes tumorigenesis, invasion and metastasis of colorectal cancer cells by transcriptional activation of FHL2. Oncotarget 2015; 6: 25402-25417. https://doi.org/10.18632/oncotarget. 4517

[11] YANG T, CAI SY, ZHANG J, LU JH, LIN C et al. Kruppellike factor 8 is a new Wnt/beta-catenin signaling target gene and regulator in hepatocellular carcinoma. PLoS One 2012; 7: e39668. https://doi.org/10.1371/journal.pone.0039668

[12] LU H, HU L, YU L, WANG X, URVALEK AM et al. KLF8 and FAK cooperatively enrich the active MMP14 on the cell surface required for the metastatic progression of breast cancer. Oncogene 2014; 33: 2909-2917. https://doi.org/10.1038/ onc. 2013.247
[13] CHEN XY, LO TC. Phosphorylation of a cell surface 112 $\mathrm{kDa}$ protein by an ecto-protein kinase in rat L6 myoblasts. Biochem J 1991; 279: 467-474. https://doi.org/10.1042/ bj2790467

[14] Wang X1, Zheng M, Liu G, Xia W, McKeown-Longo PJ et al. Kruppel-like factor 8 induces epithelial to mesenchymal transition and epithelial cell invasion. Cancer Res 2007; 67: 7184-7893. https://doi.org/10.1158/0008-5472.CAN-06-4729

[15] LU H, HU L, LI T, LAHIRI S, SHEN C et al. A novel role of Kruppel-like factor 8 in DNA repair in breast cancer cells. J Biol Chem 2012; 287: 43720-43729. https://doi.org/10.1074/ jbc.M112.418053

[16] WANG X, LU H, LI T, YU L, LIU G et al. Kruppel-like factor 8 promotes tumorigenic mammary stem cell induction by targeting miR-146a. Am J Cancer Res 2013; 3: 356-373.

[17] LIN F, SHEN Z, TANG LN, ZHENG SE, SUN YJ et al. KLF8 knockdown suppresses proliferation and invasion in human osteosarcoma cells. Mol Med Rep 2014; 9: 1613-1617. https://doi.org/10.3892/mmr.2014.2027

[18] LAHIRI SK, ZHAO J. Kruppel-like factor 8 emerges as an important regulator of cancer. Am J Transl Res 2012; 4: 357-363.

[19] THIERY JP, ACLOQUE H, HUANG RY, NIETO MA. Nieto, Epithelial-mesenchymal transitions in development and disease. Cell 2009; 139: 871-890. https://doi.org/10.1016/j. cell.2009.11.007

[20] TAKEBE N, WARREN RQ, IVY SP. Breast cancer growth and metastasis: interplay between cancer stem cells, embryonic signaling pathways and epithelial-to-mesenchymal transition. Breast Cancer Res 2011; 13: 211. https://doi. org/10.1186/bcr2876

[21] ZHANG L, GUO X, ZHANG L, YANG F, QIN L et al. SLC34A2 regulates miR-25-Gsk3beta signaling pathway to affect tumor progression in gastric cancer stem cell-like cells. Mol Carcinog 2018; 57: 440-450. https://doi.org/10.1002/ mc. 22768

[22] WANG T, WANG D, ZHANG L, YANG P, WANG J et al. The TGFbeta-miR-499a-SHKBP1 pathway induces resistance to EGFR inhibitors in osteosarcoma cancer stem celllike cells. J Exp Clin Cancer Res 2019; 38: 226. https://doi. org/10.1186/s13046-019-1195-y

[23] DENG Y, LUAN F, ZENG L, ZHANG Y, MA K. MiR-429 suppresses the progression and metastasis of osteosarcoma by targeting ZEB1. EXCLI J 2017; 16: 618-627. https://doi. org/10.17179/excli2017-258

[24] LIU X, LIU Y, WU S, SHI X, LI L et al. Tumor-suppressing effects of miR-429 on human osteosarcoma. Cell Biochem Biophys 2014; 70: 215-224. https://doi.org/10.1007/s12013014-9885-8

[25] MA S, TANG KH, CHAN YP, LEE TK, KWAN PS et al. miR-130b Promotes CD133(+) liver tumor-initiating cell growth and self-renewal via tumor protein 53-induced nuclear protein 1. Cell Stem Cell 2010; 7: 694-707. https://doi. org/10.1016/j.stem.2010.11.010

[26] SHEN YN, HE HG, SHI Y, CAO J, YUAN JY et al. Kruppellike factor 8 promotes cancer stem cell-like traits in hepatocellular carcinoma through Wnt/beta-catenin signalling. Mol Carcinog 2017; 56: 751-760. https://doi.org/10.1002/ mc.22532 
[27] ZHAO J, BIAN ZC, YEE K, CHEN BP, CHIEN S et al. Identification of transcription factor KLF8 as a downstream target of focal adhesion kinase in its regulation of cyclin D1 and cell cycle progression. Mol Cell 2003; 11: 1503-1515. https:// doi.org/10.1016/s1097-2765(03)00179-5

[28] AVIZIENYTE E, FRAME MC. Src and FAK signalling controls adhesion fate and the epithelial-to-mesenchymal transition. Curr Opin Cell Biol 2005; 17: 542-547. https://doi. org/10.1016/j.ceb.2005.08.007

[29] NAKAMURA K, YANO H, SCHAEFER E, SABE H. Different modes and qualities of tyrosine phosphorylation of Fak and Pyk2 during epithelial-mesenchymal transdifferentiation and cell migration: analysis of specific phosphorylation events using site-directed antibodies. Oncogene 2001; 20: 2626-2635. https://doi.org/10.1038/sj.onc.1204359
[30] SARKAR A, HOCHEDLINGER K. The sox family of transcription factors: versatile regulators of stem and progenitor cell fate. Cell Stem Cell 2013; 12: 15-30. https://doi. org/10.1016/j.stem.2012.12.007

[31] WANG J, RAO S, CHU J, SHEN X, LEVASSEUR DN et al. A protein interaction network for pluripotency of embryonic stem cells. Nature 2006; 444: 364-368. https://doi. org/10.1038/nature05284

[32] WEINA K, UTIKAL J. SOX2 and cancer: current research and its implications in the clinic. Clin Transl Med 2014; 3:19. https://doi.org/10.1186/2001-1326-3-19

[33] BASU-ROY U, SEO E, RAMANATHAPURAM L, RAPP TB, PERRY JA et al. Sox2 maintains self renewal of tumorinitiating cells in osteosarcomas. Oncogene 2012; 31: 22702282. https://doi.org/10.1038/onc.2011.405 


\section{KLF8 promotes cancer stem cell-like phenotypes in osteosarcoma through miR-429-SOX2 signaling}

L. ZHANG, P. YANG, Q. LIU, J. WANG, F. YAN, L. DUAN, F. LIN*

\section{Supplementary Information}

Supplementary Table S1. Expression of CD133, KLF8 and miR-429 in osteosarcoma samples.

\begin{tabular}{|c|c|c|c|c|c|c|c|}
\hline Case & Age & TNM & $\begin{array}{c}\text { Clinical } \\
\text { stage }\end{array}$ & $\begin{array}{l}\text { Percentage of } \\
\text { CD133+cells }\end{array}$ & $\begin{array}{l}{ }^{\text {a Normalized KLF8 }} \\
\text { amount } 2^{-\Delta \Delta \mathrm{Ct}}\end{array}$ & $\begin{array}{c}{ }^{\text {a Normalized miR-429 }} \\
\text { amount } 2^{-\Delta \Delta C t}\end{array}$ & $\begin{array}{c}\text { Sphere } \\
\text { formation }\end{array}$ \\
\hline 1 & 56 & T1N0M0 & IA & $0.07 \%$ & 4.3600 & 0.3027 & Yes \\
\hline 2 & 61 & T2N0M0 & IB & $0.03 \%$ & 4.6023 & 0.2964 & Yes \\
\hline 3 & 44 & T1N1M0 & IIA & $1.06 \%$ & 2.1854 & 0.1392 & No \\
\hline 4 & 57 & T2N1M0 & IIA & $1.83 \%$ & 3.0938 & 0.4336 & Yes \\
\hline 5 & 49 & T2N2M0 & IIB & $1.20 \%$ & 2.6927 & 0.2578 & Yes \\
\hline 6 & 67 & T3N0M0 & IIIA & $1.38 \%$ & 3.2247 & 0.1937 & Yes \\
\hline 7 & 58 & T3N3M1 & IV & $0.95 \%$ & 5.3219 & 0.2026 & Yes \\
\hline 8 & 59 & T2N2M0 & IIIA & $0.84 \%$ & 4.3963 & 0.1962 & Yes \\
\hline 9 & 51 & T2N0M0 & IB & $0.69 \%$ & 5.2014 & 0.3525 & No \\
\hline 10 & 72 & T2N3M0 & IIIB & $1.81 \%$ & 4.0390 & 0.4669 & Yes \\
\hline
\end{tabular}

${ }^{a}$ Relative quantification was performed by the $2^{-\Delta \Delta C t}$ method with the adjacent osteosarcoma tissue sample as a calibrator. Data show the means from three independent analyses. Every independent analysis was carried out after the RNA extraction step. Total RNA was poly-A tailed, reverse transcript, and then real-time PCR tested. $\Delta \mathrm{C}_{\mathrm{T}}$ obtained from real-time PCR was subject to paired t-test $\left(\Delta \mathrm{C}_{\mathrm{T}}=\mathrm{C}_{\mathrm{T} \text { KLFs }}-\mathrm{C}_{\mathrm{TGAPDH}}\right)$. $\Delta \mathrm{C}_{\mathrm{T}}$ obtained from real-time PCR was subject to paired t-test $\left(\Delta \mathrm{C}_{\mathrm{T}}=\mathrm{C}_{\mathrm{T} \text { miR-429 }}-\mathrm{C}_{\mathrm{T} \text { U6 }}\right)$. The expression levels of KLF8 in tumor tissues were significantly higher than normal tissues ( $<<0.01$ ). The expression levels of miR-429 in tumor tissues were significantly lower than normal tissues $(p<0.01)$. 